\title{
Identification of Structural Loss Factor from Spatially Distributed Measurements on Beams with Viscoelastic Layer *
}

\author{
Michał Rak ${ }^{\mathrm{a}}$, Mohamed Ichchou ${ }^{\mathrm{b}, *}$, Jan Holnicki-Szulc ${ }^{\mathrm{a}}$ \\ ${ }^{a}$ Institute of Fundamental Technological Research, Polish Academy of Sciences, Świętokrzyska 21, 00-049 Warsaw, Poland \\ ${ }^{\mathrm{b}}$ Laboratoire de Tribologie et Dynamique des Systèmes, Ecole Centrale de Lyon, 36 avenue Guy de Collongue, F69134 \\ ECULLY Cedex, France
}

\begin{abstract}
The problem of estimation of a structural loss factor for a beam covered with a viscoelastic layer is addressed in the paper. Two estimation methods based on analytical models for wave propagation in viscoelastic homogenous beams are tested. The methods use different theoretical solutions for spatial distribution of a wave field in the beam. The solutions depend on a complex wave number and frequency. At each frequency within an investigated range the wave number, for which model predictions best approximate experimental response, is found. Structural loss factor is calculated based on the identified value of wave number. Experimental data are measured in a cantilever beam test. For verification purposes the obtained values of loss factor are compared with the results of Oberst test. The presented methods enable determination of loss factor for arbitrary discrete frequencies. They provide an alternative to modal techniques which estimate only values of the parameter corresponding to resonant frequencies. doi:
\end{abstract}

\section{Introduction}

The problem of estimation of a structural loss factor for beams with viscoelastic layer is addressed in the paper. Two methods, developed by Berthaut et al. [1,2] and McDaniel et al. [3,4], are used independently to identify the structural loss factor for a cantilever beam subjected to flexural vibrations. Oberst [5,6], who as a first investigated the problem, considered thin metal strips covered with an adhesive viscoelastic tape. He derived an analytical relation between a loss factor of the composite and of the viscoelastic layer using kinematic and physical formulas of the classical theory of beams. Analogical expressions, but for a plate covered with viscoelastic sheet placed under elastic constraining layer were given by Kerwin et al. [7,8].

Oberst has also developed an experimental procedure for measuring the structural loss factor. Half-value bandwidth method, as it is referred to in literature, exploits a relation between shape of a resonance peak and a value of the loss factor. It still serves as a basic tool for estimation of the loss factor but it suffers all inherent disadvantages of a modal formulation. Due to a drastic increase of a modal density in higher frequencies, observed in all structures, the approach is limited to the low band covering first few modes of vibrations. Only values of the loss factor, corresponding to natural frequencies, can be estimated by means of the test. Moreover, the method is sensitive to imperfections in experimental boundary conditions.

The two methods mentioned at the beginning, namely Inhomogeneous Wave Correlation, devised by Berthaut et al. $[1,2]$, and the one developed by McDaniel et al. [3,4], stem from the need to overcome the limitations met in the modal description. They are based on the hypothesis that the spatial terms which contribute to the solution of free vibration problem, correspond not only to natural frequencies but to all frequencies. Berthaut

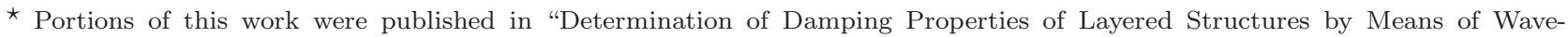
Propagation-Based Methods", Mechanics 24 (AH University of Science and Technology Press, Cracow, 2005) 140-143.

* Corresponding author.

Email addresses: mrak@ippt.gov.pl (Michał Rak), mohamed.ichchou@ec-lyon.fr (Mohamed Ichchou), holnicki@ippt.gov.pl (Jan Holnicki-Szulc).
} 
et al. [2] have successfully applied their method to the identification of dispersion curves in structurally orthotropic plates. The results presented in [2] indicate that the identification procedure is dependent neither on boundary conditions nor on load location. Berthaut [1] has also applied the method to a numerical model of a beam subjected to bending. However, the simulation has not been supported by experiment. In this work Inhomogeneous Wave Correlation ${ }^{1}$ has been employed to estimate complex wave number for flexural waves propagating in viscoelastic beams. The underlying equations have been used to derive a relation between the wave number and loss factor.

In both discussed methods Fourier transform is used instead of a classical technique of separation of variables to obtain an equation describing distribution of a wave field in the beam. However, while McDaniel et al. employ general solution of the equation, which is composed of four terms, Berthaut [1] postulates using only one of the terms, related to an incident wave, and discarding others, corresponding to evanescent waves. The question arises if such a simplification is allowed even if only measurements done away from boundaries are compared with the theoretical predictions. This issue is addressed in the paper. To validate and compare the methods experimental investigations have been conducted. The measurements done in spatially distributed points lying along the axis of a tested beam have been Fourier transformed. Since phase difference between the responses is crucial for correct identification of the wave field a transfer function has been calculated.

The results presented below show that the solution assumed in IWC does not allow for a proper description of the wave field observed in the experiment. It has been investigated if the complex wave number determined by IWC could be used as a starting value in the identification algorithm employed by McDaniel et al. Due to a poor performance of the former method the idea was abandoned. Influence of measurement noise on the obtained results has been taken into account by calculation of coherence function and its introduction into the identification procedure. As the theoretical description of the wave field proposed in IWC lacks terms corresponding to a nearfield, it is investigated how neglecting of the measurements done in the vicinity of boundaries affects estimates of the wave number. The paper presents values of wave number and loss factor, identified from the test conducted on a steel sample covered with a layer of a viscoelastic material, subjected to random loading. Middle and high frequency range is of interest to the authors. For comparative reasons values of the loss factor determined in Oberst test are also demonstrated.

This work was motivated by the need to overcome problems encountered in finite element modelling of multilayer structures including viscoelastic sheets. Dynamic analysis of such systems is troublesome, mainly due to damping effects, which have to be taken into account. Defining each layer separately complicates the problem. It requires knowledge of viscoelastic parameters, which vary depending on an applied model of the material. Moreover, some additional assumptions have to be made about properties of adhesive films so they could be neglected. Using loss factor formulation presented below enables one to avoid these difficulties. From viewpoint of a damping characteristics the structure is then modelled as a homogeneous body. However, it needs to be remembered that this approach is valid only for the cases when the finite element model undergoes the mode of deformation identical with that induced in an experiment. It means that if $\mathrm{FE}$ model of the beam tested in the discussed study was created and a numerical analysis was performed, the reliable results would be obtained only if the model was subjected exclusively to bending.

Section 2 shows theoretical bases of IWC and McDaniel method. A notion of loss factor is introduced. Experimental procedure is described in section 3. The paper concludes with the discussion of results and final remarks.

\section{Theoretical Background}

Either Berthaut and McDaniel use linear viscoelastic model of material, derived from Boltzmann superposition principle

$$
\boldsymbol{\sigma}(t)=\int_{-\infty}^{t} \boldsymbol{\psi}(t-\tau) \frac{\partial \boldsymbol{\varepsilon}(\tau)}{\partial \tau} \mathrm{d} \tau .
$$

The term linear refers to a property of the constitutive equation understood as a relation between stress $\boldsymbol{\sigma}$, strain $\varepsilon$ and time $t$. Relaxation function, denoted by $\psi$, describes how stress changes under constant strain. If, for the mathematical simplicity, a harmonic variation of strain is assumed ${ }^{2}$

\footnotetext{
1 the acronym IWC will be used to refer to Inhomogeneous Wave Correlation in the following text

2 equation (2) has no physical meaning. It merely expresses the fact that all the solutions obtained hereinafter are valid for both real and imaginary part of Eq. (2). It is true since the following analysis operates only on linear equations. Limitation of allowed strain functions only to these represented by Eq. (2) does not affect the generality of the derivation because arbitrary variations of strain that occur over a finite duration can be approximated by Fourier series, which components are identical with already mentioned real and imaginary part of Eq. (2).
} 


$$
\varepsilon(t)=\varepsilon_{0} \mathrm{e}^{\mathrm{i} \omega t},
$$

then on substitution of Eq. (2) into Eq. (1) and taking $s=t-\tau$, one gets

$$
\boldsymbol{\sigma}(t)=\mathrm{i} \omega \varepsilon_{0} \mathrm{e}^{\mathrm{i} \omega t} \int_{0}^{\infty} \boldsymbol{\psi}(s) \mathrm{e}^{-\mathrm{i} \omega s} \mathrm{~d} s .
$$

Please, notice that the integral in Eq. (3) expresses Laplace transform of function $\psi(s)$,

$$
\Psi(\mathrm{i} \omega)=\mathcal{L}\{\boldsymbol{\psi}(s)\}=\int_{0}^{\infty} \boldsymbol{\psi}(s) \mathrm{e}^{-\mathrm{i} \omega s} \mathrm{~d} s .
$$

Now, if Eq. (2) is taken into account and time variable is omitted, Eq. (3) becomes

$$
\boldsymbol{\sigma}=\mathbf{E}(\mathrm{i} \omega) \boldsymbol{\varepsilon}
$$

where $\mathbf{E}$, defined by the relation

$$
\mathbf{E}(\mathrm{i} \omega)=\mathrm{i} \omega \Psi(\mathrm{i} \omega)
$$

is called either complex elastic modulus [9] or complex modulus of elasticity [10,13], thanks to the resemblance between Eq. (5) and the mathematical formula expressing Hook's law for the uniaxial case [11]. Some authors refer to it simply as complex modulus $[12, ?, ?]$. In the literature the complex modulus is often decomposed into real and imaginary part,

$$
\mathbf{E}=\mathbf{E}^{*}+\mathrm{i} \mathbf{E}^{\bullet}
$$

that are named storage and loss modulus of elasticity, respectively [13]. Another popular notation includes loss factor $^{3} \eta$

$$
\mathbf{E}=\mathbf{E}^{*}(1-\mathrm{i} \eta)
$$

where

$$
\eta=-\frac{\mathbf{E}^{\bullet}}{\mathbf{E}^{*}}
$$

To express the loss factor in terms of the material function $\Psi$ it is enough to compare Eqs. (6) and (8)

$$
\eta=\frac{\operatorname{Re}(\Psi(\mathrm{i} \omega))}{\operatorname{Im}(\Psi(\mathrm{i} \omega))}
$$

Solving a parameter identification problem of an experimentally tested object requires using an adequate theoretical model. Thus the governing equation for transverse motion of a viscoelastic beam

$$
J \int_{-\infty}^{t} \psi(t-\tau) \frac{\partial}{\partial \tau}\left(\frac{\partial^{4} w}{\partial x^{4}}\right) \mathrm{d} \tau+\rho A \frac{\partial^{2} w}{\partial t^{2}}=0
$$

is employed in the presented methods. The beam is characterised by cross section $A$, moment of inertia $J$ and density $\rho$. No distributed force is assumed. Equation (11) has been obtained with the use of Boltzmann superposition principle expressed by Eq. (1). It needs to be stressed that the derivation of Eq. (11) is not trivial. It is based on the assumption that the simple elongation of a viscoelastic fibre can be described by the Eq. (1). To prove it one needs to write Eq. (1) in a scalar form, dependent on a chosen coordinate system, and rearrange the obtained equation, remembering that some components of stress vanish.

IWC and McDaniel methods enable determination of dispersion curves in the structures under flexure. Since IWC method was originally applied to identification of a wave field in plates, plane waves

$$
w(x, y, t)=w_{0} \mathrm{e}^{\mathrm{i}(\omega t-\mathbf{k} \cdot \mathbf{x})}
$$

were of interest to Berthaut et al. The foregoing equation ${ }^{4}$ describes propagation of a wave of frequency $\omega$ in a two-dimensional medium. Scalar product $\mathbf{k} \cdot \mathbf{x}$ represents location of the wave front in a moment $t$. It can be expanded to the form

$$
\mathbf{k} \cdot \mathbf{x}=k \mathbf{n} \cdot \mathbf{x}
$$

where $k$ is a wave number, $\mathbf{n}$ is a vector normal to the plane of propagation and $\mathbf{x}$ is a position vector. The vectors have the following representation in the basis $\left\{\mathbf{e}_{i}\right\}$ of a rectangular Cartesian coordinate system

$$
\begin{gathered}
\mathbf{n}=\cos \angle\left(\mathbf{n}, \mathbf{e}_{1}\right) \mathbf{e}_{1}+\cos \angle\left(\mathbf{n}, \mathbf{e}_{2}\right) \mathbf{e}_{2}, \\
\mathbf{x}=x \mathbf{e}_{1}+y \mathbf{e}_{2} .
\end{gathered}
$$

\footnotetext{
$\overline{3}$ it is also called loss tangent

4 equation (12) may lead to confusion. It should be replaced with either $w(x, y, t)=\operatorname{Re}\left(\mathrm{w}_{0} \mathrm{e}^{\mathrm{i}(\omega t-\mathbf{k} \cdot \mathbf{x})}\right)$ or $w(x, y, t)=$ $\operatorname{Im}\left(\mathrm{w}_{0} \mathrm{e}^{\mathrm{i}(\omega \mathrm{t}-\mathbf{k} \cdot \mathbf{x})}\right)$. Both forms are correct and satisfy equations of motion derived within a linear theory of plates. However, for the sake of simplicity one maintains notation used in Eq. (12) throughout the rest of the text.
} 
If a one-directional propagation is considered, which is a common assumption in the case of beams, Eq. (12) describing the wave field stays unaltered except that one spatial variable is used instead of two. Hence, for a definite frequency $\omega$ transverse deflection of the beam is described by equation

$$
W(x)=\tilde{w}_{0} \mathrm{e}^{-\mathrm{i} k x} .
$$

It should be emphasised that from viewpoint of the classical theory of beams, Eq. (16), which underlies IWC method, is incomplete since it lacks the term representing an evanescent wave. This fact is taken into account in an identification procedure, where the measurements of lateral displacements, done in points lying close the boundaries of a sample are discarded. It is assumed in the method that propagation occurs in a dissipative medium. Thus, complex values of wave number are obtained in frequency domain. If the problem of bending waves is investigated, which is the case, a relation between wave number $k$ and loss factor $\eta$ is given by ratio of phase and group velocity, denoted by $c_{\varphi}$ and $c_{g}$, respectively:

$$
\frac{\operatorname{Im}(\mathrm{k})}{\operatorname{Re}(\mathrm{k})}=\eta \frac{c_{\varphi}}{2 c_{g}}
$$

Berthaut et al. have borrowed this equation from other work devoted to modelling of vibrating systems [?]. Since for bending waves the group velocity is twice the phase velocity, the loss factor may be expressed directly in terms of the wave number:

$$
\eta=4 \frac{\operatorname{Im}(\mathrm{k})}{\operatorname{Re}(\mathrm{k})} .
$$

This formula is approximate. To obtain an exact expression for the loss factor it is enough to substitute Eq. (16) into Eq. (11), take $s=t-\tau$ and apply Eqs. (4), (6) and (8). Then one gets

$$
\eta=\frac{\operatorname{Im}\left(\mathrm{k}^{4}\right)}{\operatorname{Re}\left(\mathrm{k}^{4}\right)} .
$$

However, as one wanted to keep the original formulation of the method, Eq. (18) has been used to calculate the loss factor. It is justified for small values of the parameter. The error caused by using Eq. (18) instead of Eq. (19) does not exceed 7 percent for the values of loss factor smaller than 0.5 and it converges to zero as the loss factor approaches zero.

Correlation between the wave field observed in an experiment and the one found from Eq. (16) for a given value of the wave number and frequency, is calculated by means of the formula:

$$
I W C(k)=\frac{\left|\sum_{i=1}^{n} \bar{W}\left(x_{i}, k\right) \widetilde{W}\left(x_{i}\right) \rho\left(x_{i}\right)\right|}{\sqrt{\sum_{i=1}^{n}\left|W\left(x_{i}, k\right)\right|^{2} \sum_{i=1}^{n}\left|\widetilde{W}\left(x_{i}\right)\right|^{2} \rho\left(x_{i}\right)}},
$$

where $\widetilde{W}\left(x_{i}\right), \rho\left(x_{i}\right)$ stand for transfer function and coherence, respectively, computed for the response captured at $x_{i}, \bar{W}$ denotes the complex conjugate of $W$, and $x_{i}$ is a location of ith measurement point. The value of $k$, which maximises the ratio in Eq. (20), is a solution of the identification problem for a specified frequency. To find the solution, the space of physically allowed values of $\operatorname{Re}(\mathrm{k})$ and $\operatorname{Im}(\mathrm{k})$ is searched through. In the implemented algorithm a unit value of amplitude $\tilde{w}_{0}$ has been taken. In general, $\tilde{w}_{0}$ can be arbitrary, because it does not contribute to Eq. $(20)^{5}$, thus it does not affect the results of the identification. Further comments concerning this method can be found in reference [1].

In their method McDaniel et al. use solution $[3,4]^{6}$

$$
W(x, \omega)=c_{1}(\omega) \mathrm{e}^{\mathrm{i} k x}+c_{2}(\omega) \mathrm{e}^{-\mathrm{i} k x}+c_{3}(\omega) \mathrm{e}^{k x}+c_{4}(\omega) \mathrm{e}^{-k x}
$$

of Fourier transformed equation (11)

$$
E^{*}(\omega)(1-i \eta(\omega)) J \frac{\partial^{4} W(x, \omega)}{\partial x^{4}}-\omega^{2} \rho A W(x, \omega)=0
$$

as a model of a wave field. Equation (22) is obtained from Eq. (11) by virtue of convolution theorem and Eqs. (6) ${ }^{7}$ and (8). In mathematical terms, the Fourier transform is correct if duration of vibration $t$ tends to infinity. This condition is always violated in an experiment. However, it can be accepted for practical applications if

\footnotetext{
5 one can easily check it inserting Eq. (16) in Eq. (20)

6 in [3] terms $\mathrm{e}^{-\mathrm{i} k x}, \mathrm{e}^{k x}$ are replaced with $\mathrm{e}^{\mathrm{i} k(L-x)}, \mathrm{e}^{-k(L-x)}$, respectively. It is motivated by numerical issues.

7 symbol $\Psi(\mathrm{i} \omega)$, which appears in Eq. (6), denotes in this case Fourier not Laplace transform of function $\psi(t)$
} 
the results of Fourier analysis for the frequencies lower than the reciprocal of measurement time are discarded. The complex wave number, that appears in Eq. (21), is expressed by the formula:

$$
k=\sqrt[4]{\frac{\rho A}{E^{*}(\omega)(1-\mathrm{i} \eta(\omega)) J} \omega^{2}} .
$$

If the theoretical model is correct, equation (21) should be satisfied for every measured response. Thus, a set of equations written in a matrix form for a given frequency $\omega$

$$
\left[\begin{array}{c}
W\left(x_{1}\right) \\
\cdots \\
W\left(x_{i}\right) \\
\cdots \\
W\left(x_{n}\right)
\end{array}\right]=\left[\begin{array}{cccc}
\mathrm{e}^{\mathrm{i} k x_{1}} & \mathrm{e}^{-\mathrm{i} k x_{1}} & \mathrm{e}^{k x_{1}} & \mathrm{e}^{-k x_{1}} \\
\cdots & \cdots & \cdots & \cdots \\
\mathrm{e}^{\mathrm{i} k x_{i}} & \mathrm{e}^{-\mathrm{i} k x_{i}} & \mathrm{e}^{k x_{i}} & \mathrm{e}^{-k x_{i}} \\
\cdots & \cdots & \cdots & \cdots \\
\mathrm{e}^{\mathrm{i} k x_{n}} & \mathrm{e}^{-\mathrm{i} k x_{n}} & \mathrm{e}^{k x_{n}} & \mathrm{e}^{-k x_{n}}
\end{array}\right]\left[\begin{array}{c}
c_{1} \\
c_{2} \\
c_{3} \\
c_{4}
\end{array}\right]
$$

ought hold, where $x_{i}, i=1, \ldots, n$ denotes a location of ith measurement point and $W\left(x_{i}, \omega\right)$ is a response obtained at $x_{i}$. Unknown constants $c_{j}, j=1, \ldots, 4$ are determined by means of the least square method for each complex value $k$ assumed in consecutive loops of an optimisation procedure. The original objective function, proposed by McDaniel et al., has been modified. Now it contains the coherence function $\rho$ :

$$
\varepsilon(k)=\frac{\sqrt{\sum_{i=1}^{n}\left|W\left(x_{i}, k\right)-\widetilde{W}\left(x_{i}\right)\right|^{2} \rho\left(x_{i}\right)}}{\sqrt{\sum_{i=1}^{n}\left|\widetilde{W}\left(x_{i}\right)\right|^{2} \rho\left(x_{i}\right)}}
$$

where $\widetilde{W}\left(x_{i}\right)$ denotes an experimentally obtained response. Nelder-Mead simplex algorithm [14] has been employed to speed up a search of minimum. The loss factor is calculated from Eq. (23):

$$
\eta=\frac{\operatorname{Im}\left(\mathrm{k}^{4}\right)}{\operatorname{Re}\left(\mathrm{k}^{4}\right)}
$$

where $k$ minimising Eq. (25) is used. Please notice that Eq. (26) is identical with Eq. (19). It is obvious since both relations are derived from Eq. (11).

\section{Experimental Validation}

The experimental set-up for estimation of loss factor is shown in Fig. 1. Steel sample of dimensions 0.27 $\mathrm{m} \times 0.02 \mathrm{~m} \times 0.001 \mathrm{~m}$ covered with viscoelastic material manufactured by RIETER France was tested. Clamping was realized by means of a massive vice. An excitation was applied at the free end of the beam. Gearing\&Watson V4 shaker with Bruel\&Kjaer 8200 force charge mounted on its head, was used to provide random loading of the sample. The shaker was driven by a white noise generator. Polytec OFV 350 laser vibrometer measured velocity in 26 points spaced $0.01 \mathrm{~m}$ apart along length of the beam. Data was acquired using Bruel\&Kjaer Pulse multi-analyser system. Signal coming from the force charge was used as a reference one in FFT analysis. Frequency dependence of real and imaginary part of wave number obtained for both presented methods is shown in Figs. 2 and 3, respectively. Frequencies from $40 \mathrm{~Hz}$ to $6400 \mathrm{~Hz}$ are displayed in the plot. The upper frequency is a compromise between limitations of FFT analysis and the requirement of not exceeding Nyquist frequency. The last condition has to be satisfied if measurement points are evenly spaced, which is the case. However, as it has been demonstrated by McDaniel et al. [4], violation of the requirement is possible for irregular arrangement of the points. Very high level of noise, recorded below $40 \mathrm{~Hz}$, reflected in low values of coherence, leads to erroneous results in the range, namely real part of wave number becomes negative and loss factor takes values lying outside an interval $\langle 0,1\rangle$. Envelope of coherence calculated for all measurement points is shown in Fig. 4. Shape of the curves plotted in Fig. 2, representing real part of wave number, agrees very well with the one predicted theoretically for either conservative system and system with hysteretic model of damping applied, within the whole frequency range. Both methods produce almost identical results. However, evident differences, particularly in lower frequencies, are observed in imaginary part of wave number, as it is shown in Fig. 3. Curves representing complex wave number determined by means of IWC method, have been obtained by use of measurement data acquired in 20 points with exclusion of 6 others, lying closest to the ends of the sample. The incomplete set of data has been used on account of the fact that theoretical solution assumed in 


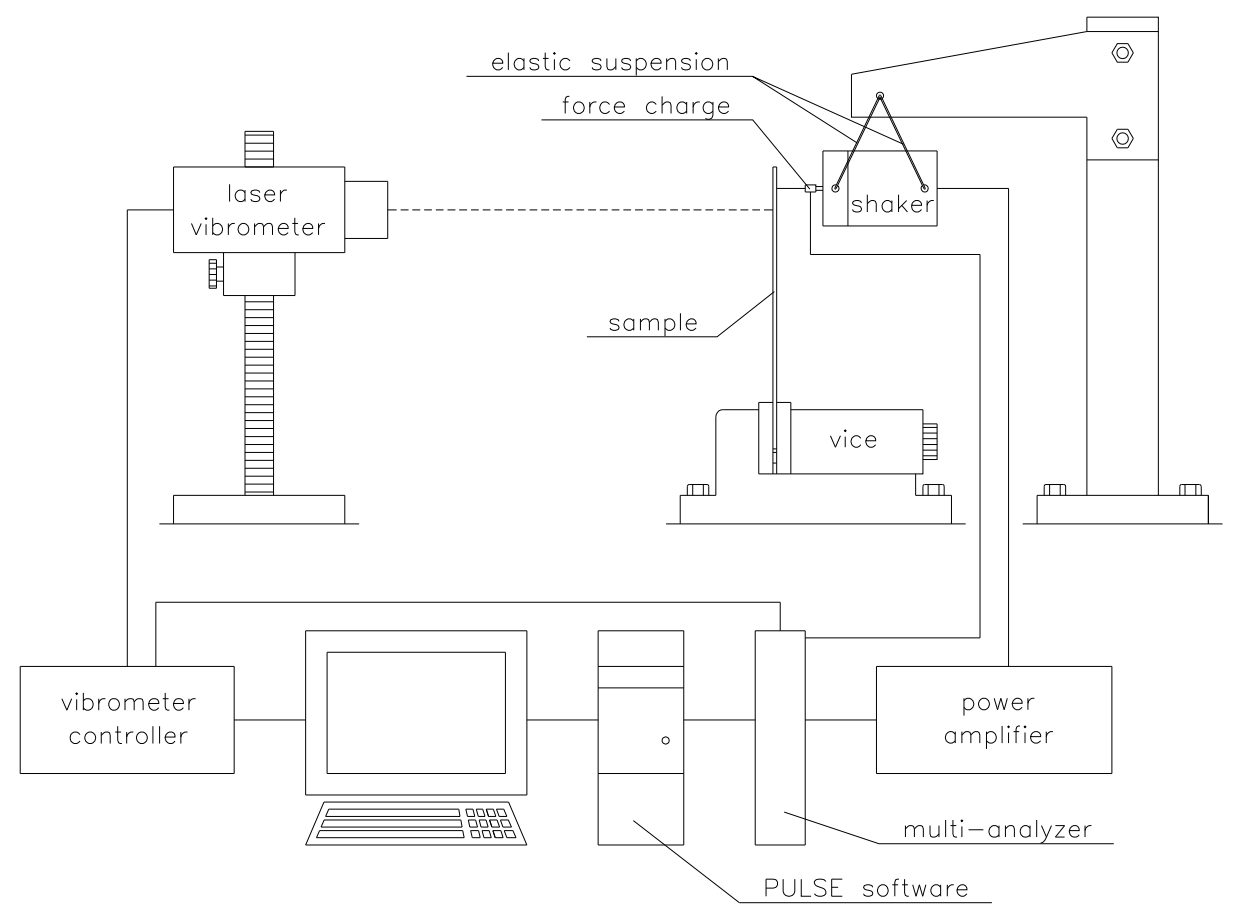

Fig. 1. Experimental set-up for identification of the complex wave number on the basis of bending wave propagation tests.

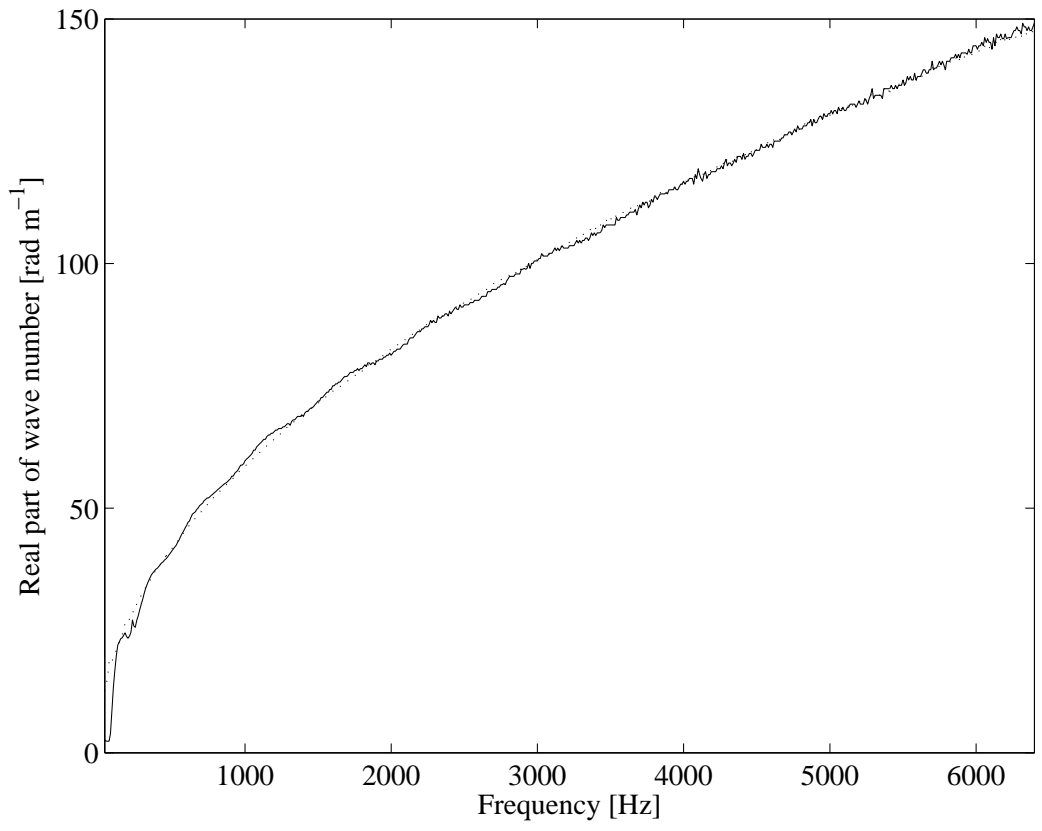

Fig. 2. Real part of wave number versus frequency. Values estimated by: — IWC method, … McDaniel method.

the method well describes the wave field existing away from boundaries. Numerical tests conducted for onedimensional wave field [1] show that IWC method returns better estimates of imaginary part of wave number if responses measured in the vicinity of boundaries are discarded. However, this effect is observed only in the case when modal solution of Eq. (11) is used to generate the wave field and it disappears if a wave solution is employed. No relevant differences in values of a real part of the wave number obtained for all mentioned cases are reported. Results of the numerical tests have been verified experimentally. Curves depicting frequency variation of real and imaginary part of the wave number determined by use of IWC method, presented in Figs. 2 and 3, are set together in a common graph marked as Fig. 5 and overlapped by respective curves obtained for complete set of measurements. The error functions defined in both methods depend on coherence. The main 


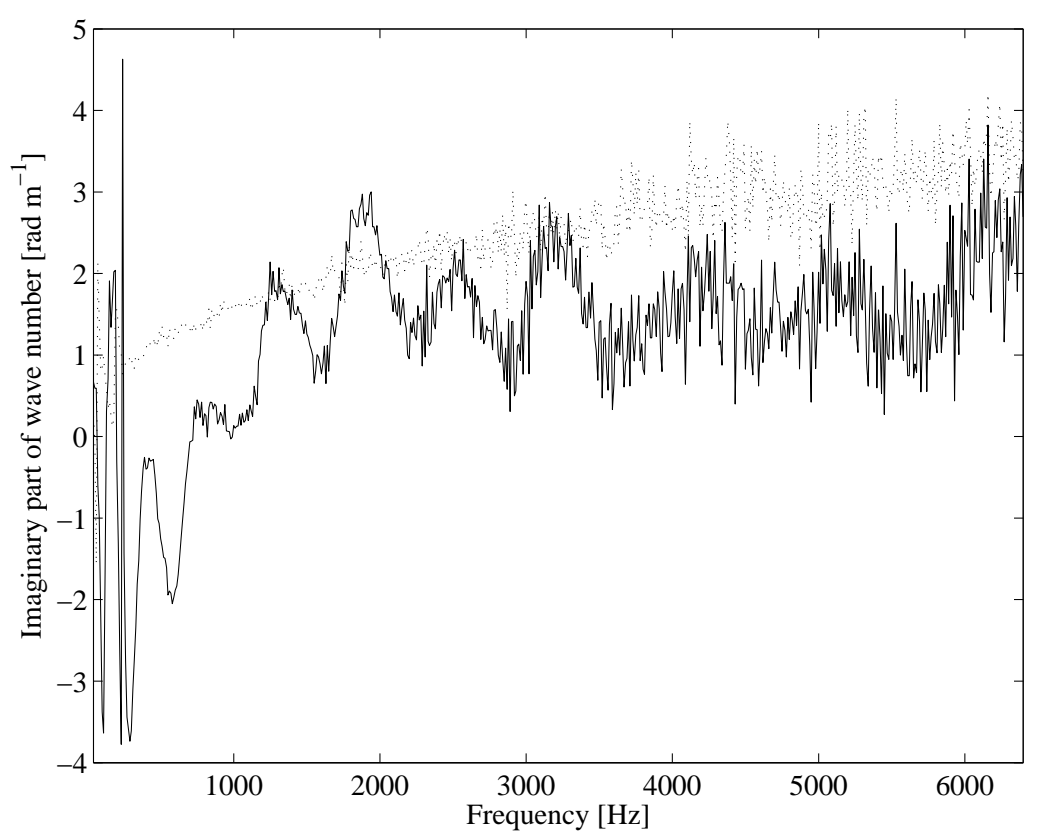

Fig. 3. Imaginary part of wave number versus frequency. Values estimated by: — IWC method, ..... McDaniel method.

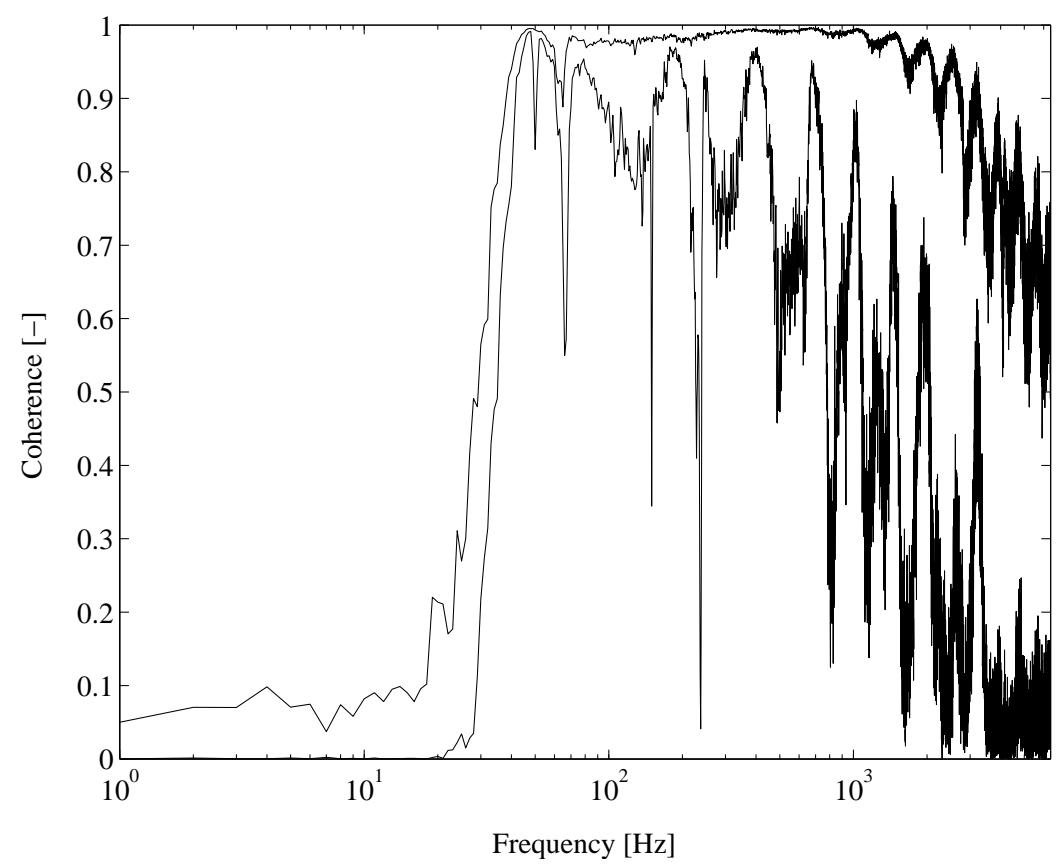

Fig. 4. Envelope of coherence function calculated for all measurement points.

reason for introducing this parameter is to eliminate the influence of measurement noise on the results of the identification procedure. Figs. 6 and 7 show differences in the returned values of real and imaginary part of wave number between the cases when coherence is taken into account and when it is neglected. Absolute differences, expressed in percents of values of the respective parameters obtained with the use of coherence, are plotted. Logarithmic scale is used for ordinate axis. Gaps in the curves depicting differences in real and imaginary part of the wave number returned by IWC method correspond to the frequencies at which no difference exists. It results in zero value, having no finite representation in logarithmic scale. From Figs. 6 and 7 it is clearly seen that unlike IWC method, McDaniel method is almost insensitive to measurement noise. It should be underlined that differences in imaginary part of wave number are on average two orders of magnitude bigger than those observed for real part. Loss factor calculated with the aid of Eqs. (18) and (26) for IWC and McDaniel method, respectively, is plotted in Fig. 8. The graph covers frequencies from $220 \mathrm{~Hz}$ to $6400 \mathrm{~Hz}$. It is based on the data presented in Figs. 2 and 3. The lower limit of the frequency range corresponds to a third resonant frequency of the tested sample. It has been discovered that precision of McDaniel method drastically decreases below 


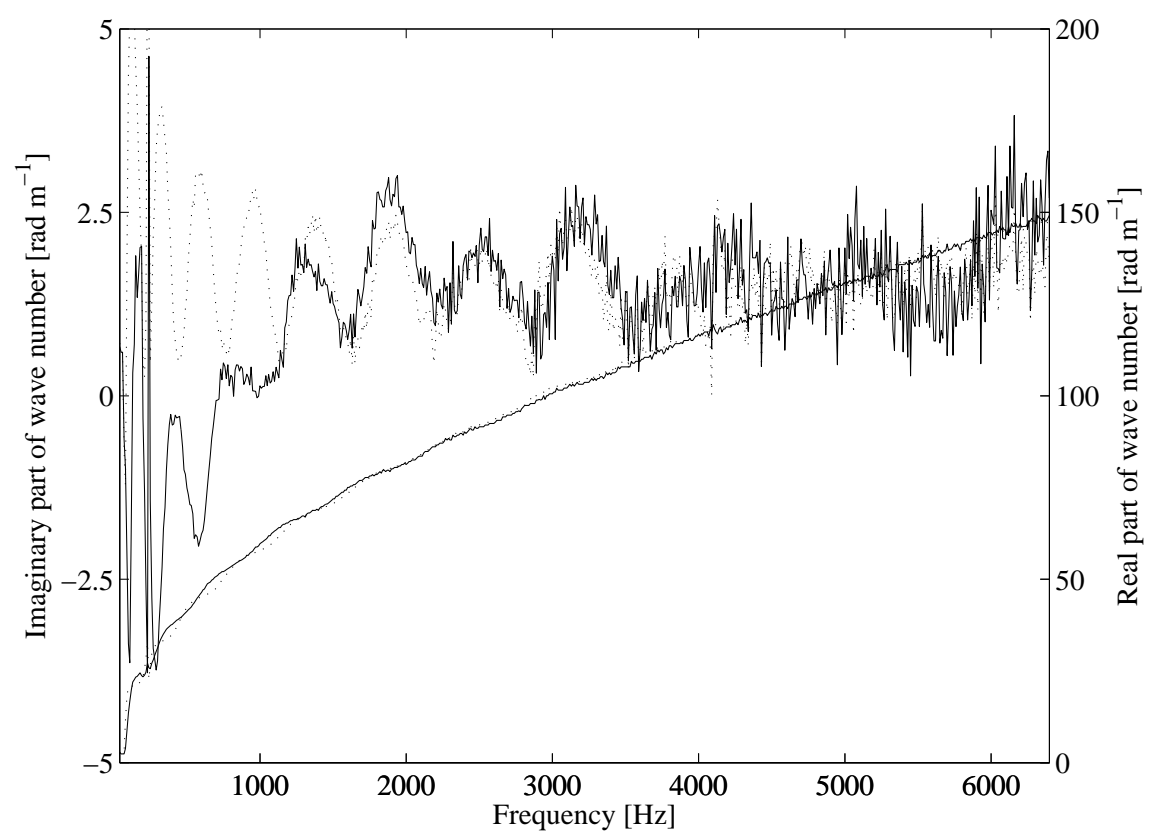

Fig. 5. Real and imaginary part of the wave number versus frequency. Values estimated by IWC method: — data from all measurement points taken into account, ..... data from measurement points lying in the vicinity of boundaries neglected.

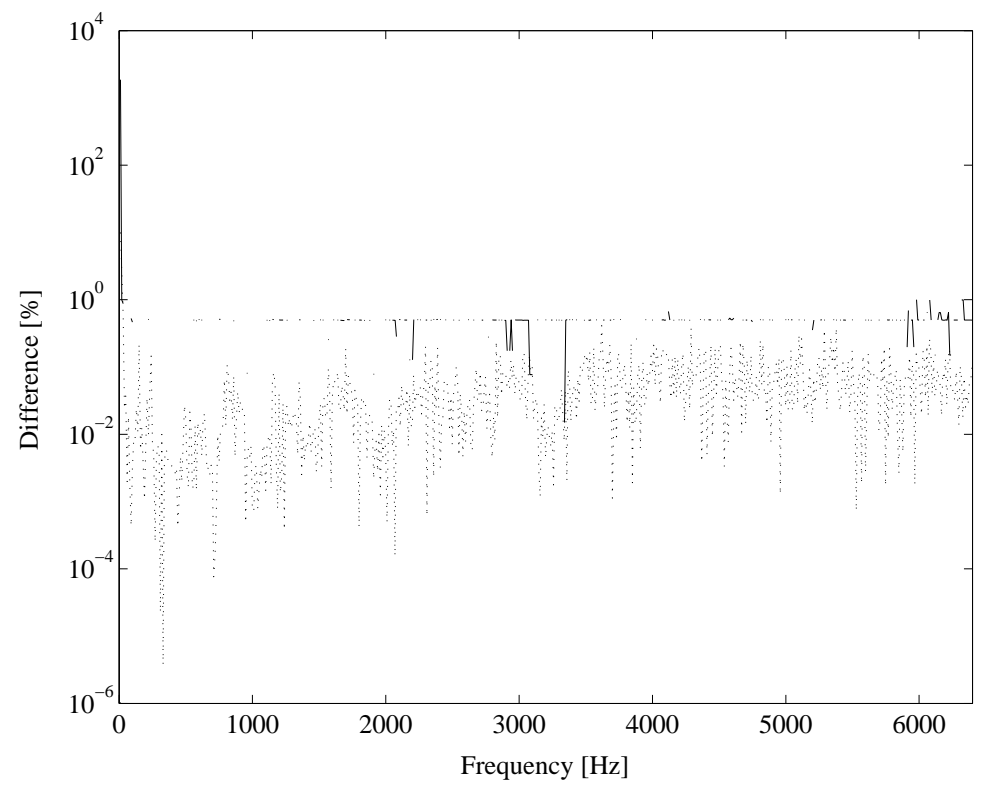

Fig. 6. Relative difference between identified values of real part of wave number for the cases when coherence appears in the objective function and when it is neglected. Real part of the wave number estimated with the use of the coherence was taken as a reference value. The difference was calculated for: - IWC method, .... McDaniel method.

that frequency in the presented case. Exceptionally high variation of imaginary part of wave number, observed in lower frequencies, confronted with a high coherence in the range and a low value of an objective function, displayed in Fig. 8, indicating good fit of the postulated solution to an experimental response, proves that the failure of the method is due to its inherent limitations. More profound theoretical analysis of the problem has been already given by McDaniel et al. [4].

Performance of IWC method needs some farther comment. Objective function employed in the method takes unit value if the theoretically predicted wave field fits ideally to the real one. The function tends to zero as the fit fails. Since IWC method searches through discretized set of admissible values of the parameters, probability of not finding global optimum is minimised provided that the discretisation is dense enough. From the last remarks and from the fact that very low values of the objective function are obtained, as is shown in Fig. 9, it is inferred that the model proposed in IWC does not describe correctly the wave field observed in the tests. This conclusion is supported by the fact that the loss factor found by the method takes negative values for 


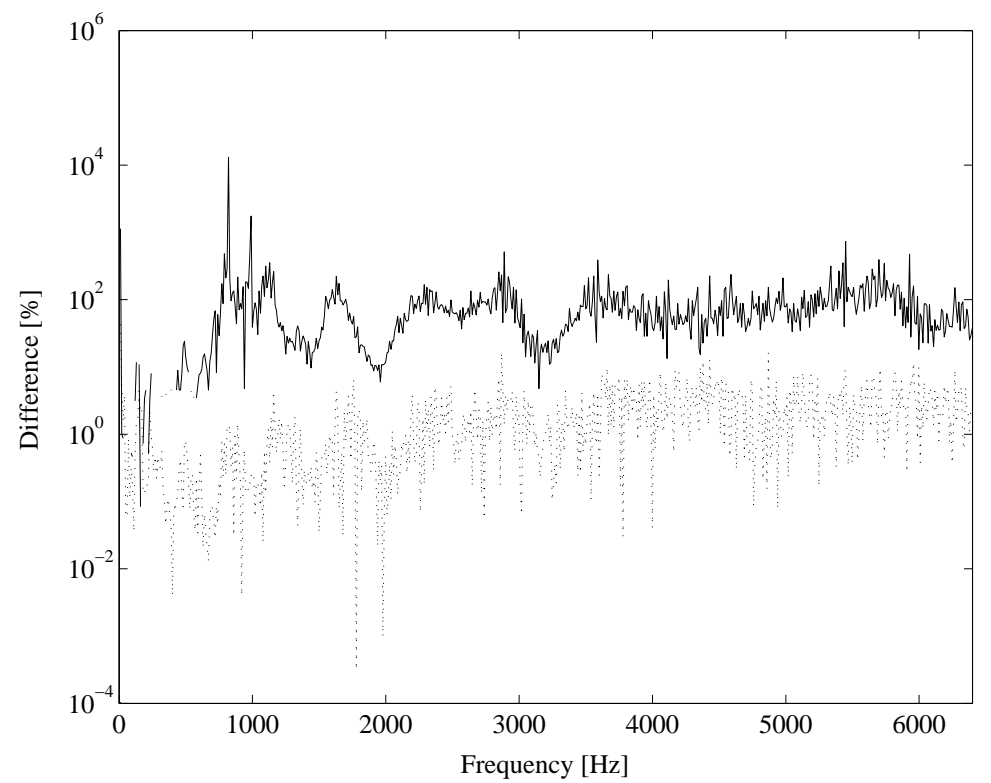

Fig. 7. Relative difference between identified values of imaginary part of wave number for the cases when coherence appears in the objective function and when it is neglected. Imaginary part of the wave number estimated with the use of the coherence was taken as a reference value. The difference was calculated for: — IWC method, ..... McDaniel method.

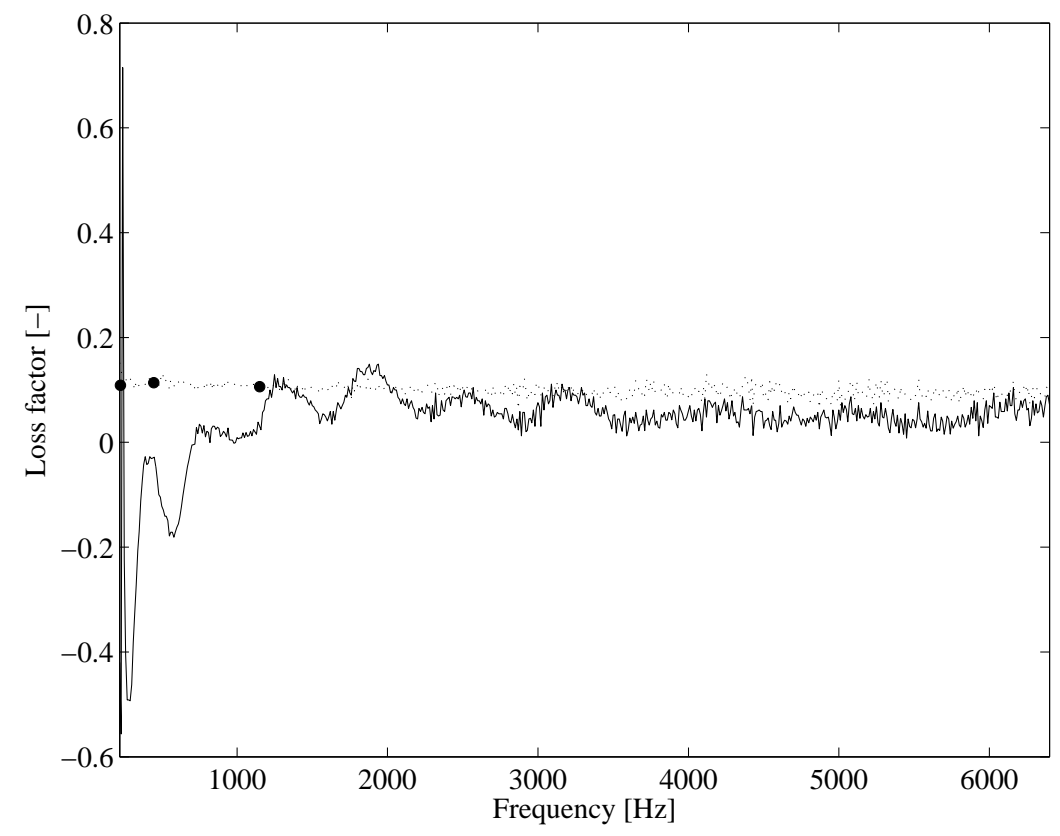

Fig. 8. Loss factor versus frequency. Values estimated by: — IWC method, … McDaniel method, • Oberst method.

lower frequencies.

Values of the loss factor obtained with the use of McDaniel method coincide with these determined in a standard Oberst test. The latter ones are depicted in Fig. 8 by black dots. The Oberst test was conducted in compliance with the standard DIN 53440. Good correlation of the results confirms the thesis that the loss factor returned by McDaniel method is correct. Fact that average value of the loss factor estimated by means of McDaniel method does not vary with frequency indicates that hysteretic model of damping applies.

\section{Conclusions}

In the presented paper the problem of estimation of the structural loss factor for the case of a cantilever elastic beam with an attached viscoelastic layer has been addressed. Two methods based on an analysis of a wave field generated in the structure have been investigated. Experimental tests have proved that the model of 


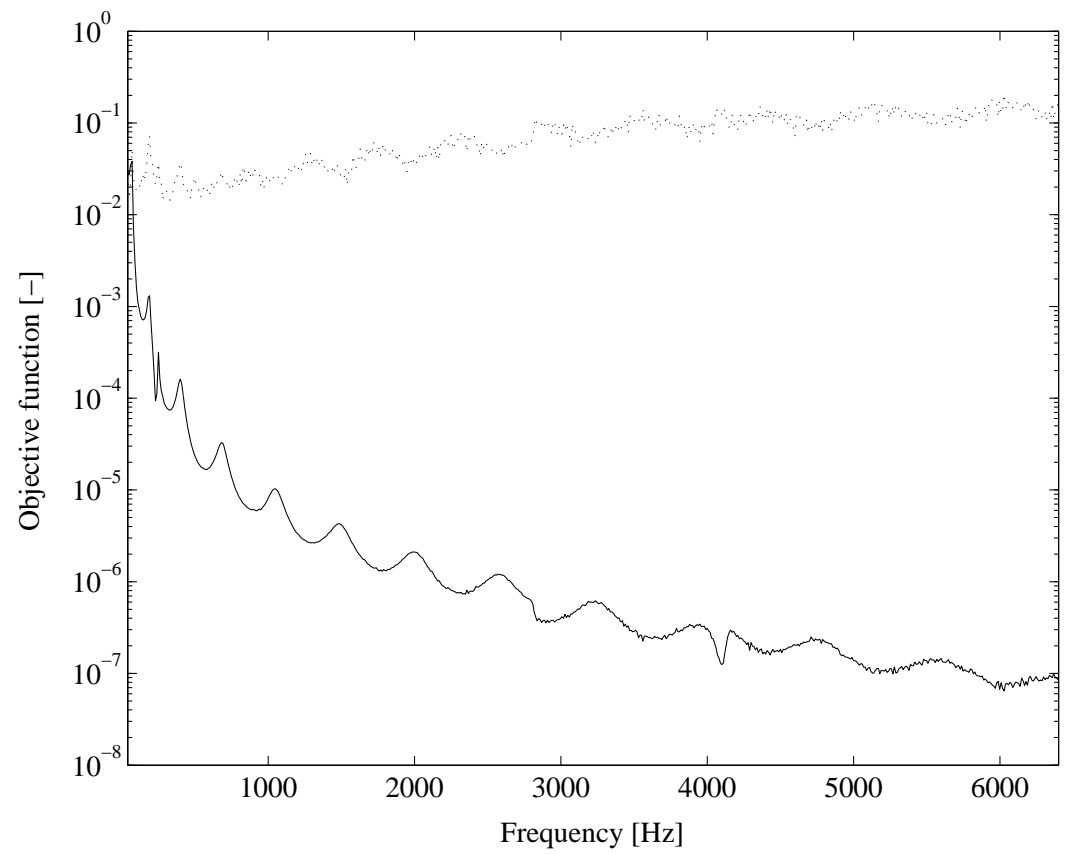

Fig. 9. Objective function versus frequency. Values obtained for: — IWC method, ..... McDaniel method.

the wave field used in IWC method is incorrect. Thus the values of the identified parameters are not reliable. It has been shown that McDaniel method enables correct estimation of the loss factor within wide frequency range. Moreover, thanks to the introduction of the loss factor notation, it allows for identification of a model of damping which occurs in the beam.

\section{Acknowledgments}

The authors wish to express their thanks to Dr. Julien Berthaut for his helpful remarks. Results of Oberst test have been published by courtesy of RIETER Automotive company, which has also provided the samples. Grateful acknowledgement is made for the support obtained from the 5FP EU project Research Training Networks "SMART SYSTEM" HPRN-CT-2002-00284.

\section{References}

[1] J. Berthaut, Contribution a l'identifcation large bande des structures anisotropes, Ph.D. thesis, Ecole Centrale de Lyon, Lyon, France, in French (2004)

[2] J. Berthaut, M. Ichchou, L. Jezequel, K-space identification of apparent structural behaviour, Journal of Sound and Vibration 280 (5) (2005) 1125-1131.

[3] J. McDaniel, W. S. Shepard Jr, Estimation of structural wave numbers from spatially sparse response measurements, Journal of the Acoustical Society of America 108 (4) (2000) 1674-1682.

[4] J. McDaniel, P. Dupont, L. Salvino, A wave-approach to estimating frequency-dependent damping under transient loading, Journal of Sound and Vibration 231 (2000) 433-449.

[5] H. Oberst, Über die dämpfung der biegeschwingungen dünner bleche durch fest haftende beläge, Acustica 2 Akustische Beihefte 4 (1952) 181-184

[6] H. Oberst, Über die dämpfung der biegeschwingungen dünner bleche durch fest haftende beläge ii, Acustica 4 Akustische Beihefte 4 (1954) 433-444.

[7] E. M. Kerwin Jr, Damping of flexural waves by a constrained visco-elastic layer, Journal of the Acoustical Society of America 31 (4) (1959) 952-962.

[8] D. Ross, E. Ungar, E. M. Kerwin Jr, Structural Damping, ASME, New York, 1959, Ch. Damping of Plate Flexural Vibrations by Means of Viscoelastic Laminae, pp. 49-87.

[9] B. Gross, Mathematical Structure of the Theories of Viscoelasticity, Hermann, Paris, 1968.

[10] L. Cremer, M. Heckl, Structure-Borne Sound, Springer-Verlag, Berlin, 1973.

[11] S. C. Hunter, Progress in Solid Mechanics, North Holland Publishing Company, 1960, Ch. Viscoelastic Waves, pp. 3-57.

[12] D. R. Bland, The Theory of Linear Viscoelasticity, Pergamon Press, Belfast, 1960.

[13] J. Ruzicka (Ed.), Structural Damping, ASME, 1959.

[14] J. Lagarias, J. Reeds, P. W. M.H. Wright, Convergence properties of the nelder-mead simplex method in low dimensions, SIAM Journal of Optimisation 9 (1) (1998) 112-147. 